\title{
An Explicit Formula for Symmetric Polynomials Related to the Eigenfunctions of Calogero-Sutherland Models ${ }^{\star}$
}

\author{
Martin $H A L L N \ddot{A} S$ \\ Department of Theoretical Physics, Albanova University Center, SE-106 91 Stockholm, Sweden \\ E-mail: hallnas@kth.se \\ URL: http://theophys.kth.se/ martin/
}

Received November 01, 2006, in final form February 05, 2007; Published online March 01, 2007

Original article is available at http://www.emis.de/journals/SIGMA/2007/037/

\begin{abstract}
We review a recent construction of an explicit analytic series representation for symmetric polynomials which up to a groundstate factor are eigenfunctions of CalogeroSutherland type models. We also indicate a generalisation of this result to polynomials which give the eigenfunctions of so-called 'deformed' Calogero-Sutherland type models.
\end{abstract}

Key words: quantum integrable systems; orthogonal polynomials; symmetric functions

2000 Mathematics Subject Classification: 81Q05; 35Q58; 33C52

\section{Introduction}

The purpose of this paper is to give a pedagogical review of a recent construction of an explicit analytic series representation for symmetric polynomials which up to a groundstate factor are eigenfunctions of Calogero-Sutherland type models. In special cases, this construction has been previously studied in $[7,11,12]$, while a detailed account of the more general results presented in this paper is in preparation [8].

To establish a context for our construction we begin this introduction by briefly discussing quantum many-body models of Calogero-Sutherland type in general and highlighting some of the distinguishing features of those models which have eigenfunctions given by polynomials. By reviewing Sutherland's original solution method for the Calogero model [23] we proceed to recall that these polynomials have a triangular structure and to discuss its importance when explicitly constructing them. We then sketch the main steps in our solution method and compare it to Sutherland's. An outline for the remainder of the paper finally concludes the introduction.

\subsection{Quantum many-body models of Calogero-Sutherland type}

A quantum many-body model of Calogero-Sutherland type is for some potential functions $V$ and $W$ defined by the Schrödinger operator

$$
H_{N}=-\sum_{j=1}^{N} \frac{\partial^{2}}{\partial x_{j}^{2}}+\sum_{j=1}^{N} V\left(x_{j}\right)+\sum_{j<k} W\left(x_{j}, x_{k}\right),
$$

where $N$ refers to the number of particles present in the system, and $x_{j}$ to their positions. As first observed by Calogero [3] and Sutherland [23] in two special cases there exist certain choices of

*This paper is a contribution to the Proceedings of the Workshop on Geometric Aspects of Integrable Systems (July 17-19, 2006, University of Coimbra, Portugal). The full collection is available at http://www.emis.de/journals/SIGMA/Coimbra2006.html 
these potential functions for which the resulting Schrödinger operator can be exactly solved. For many of these choices this is due to the fact that its eigenfunctions are given by natural manyvariable generalisations of polynomials solving a second order ordinary differential equation. This includes for example the original models of Calogero and Sutherland, whose eigenfunctions respectively correspond to the Hermite polynomials and the ordinary monomials $p_{n}=x^{n}$. We also mention Olshanetsky and Perelomov's [19] root system generalisations of these models, where the Legendre, Gegenbauer and Jacobi polynomials similarly appear; see $[1,26]$.

All of these models share a number of remarkable properties: their square integrable eigenfunctions, labelled by partitions $\lambda=\left(\lambda_{1}, \ldots, \lambda_{N}\right)$, i.e. integers $\lambda_{i}$ such that $\lambda_{1} \geq \cdots \geq \lambda_{N} \geq 0$, are all of the form

$$
\Psi_{\lambda}\left(x_{1}, \ldots, x_{N}\right)=\Psi_{0}\left(x_{1}, \ldots, x_{N}\right) P_{\lambda}\left(z\left(x_{1}\right), \ldots, z\left(x_{N}\right)\right)
$$

with particular symmetric polynomials $P_{\lambda}$ and a ground state $\Psi_{0}$ which always is of the product form

$$
\Psi_{0}\left(x_{1}, \ldots, x_{N}\right)=\prod_{j=1}^{N} \psi_{0}\left(x_{j}\right) \prod_{j<k}\left(z\left(x_{k}\right)-z\left(x_{j}\right)\right)^{\kappa},
$$

where the function $z$ is fixed by the choice of potential function $V$, and $\psi_{0}$ is the ground state of the one-body model obtained by setting $N=1$ in (1). The corresponding eigenvalues are in addition of a very simple form and can be written down explicitly. In the remainder of this paper we will refer to the polynomials $P_{\lambda}$ as reduced eigenfunctions of the corresponding Schrödinger operator (1) and our aim is to explain an explicit analytic series representation for them, obtained in [8].

\subsection{Triangular structures and Sutherland's solution method}

If we conjugate the Schrödinger operator (1) by its groundstate (3), and subtract the corresponding eigenvalue $E_{0}$, we obtain the differential operator

$$
\tilde{H}_{N}:=\Psi_{0}^{-1}\left(H-E_{0}\right) \Psi_{0}=-\sum_{j=1}^{N} \frac{\partial^{2}}{\partial x_{j}^{2}}-2 \sum_{j=1}^{N} \Psi_{0}^{-1} \frac{\partial \Psi_{0}}{\partial x_{j}} \frac{\partial}{\partial x_{j}},
$$

which has the symmetric polynomials $P_{\lambda}$ as eigenfunctions. It was observed already by Sutherland [24] that a key property in their construction is that this differential operator can be consistently restricted to certain finite dimensional subspaces of the symmetric polynomials, on which it can be represented by a finite dimensional triangular matrix. This reduces the problem of constructing the reduced eigenfunctions of the Schrödinger operator (1) to that of diagonalising a finite dimensional triangular matrix.

To make this more precise we now present a slight modification of Sutherland's original argument for the so-called Calogero model, defined by the Schrödinger operator (1) for $V(x)=$ $x^{2}$ and $W(x, y)=2 \kappa(\kappa-1)(x-y)^{-2}$ with $\kappa>0$, i.e.,

$$
H_{N}=-\sum_{j=1}^{N} \frac{\partial^{2}}{\partial x_{j}^{2}}+\sum_{j=1}^{N} x_{j}^{2}+2 \kappa(\kappa-1) \sum_{j<k} \frac{1}{\left(x_{j}-x_{k}\right)^{2}} .
$$

Note that we without loss of generality have set the harmonic oscillator frequency $\omega>0$ to 1 : it can be introduced by scaling $x_{j} \rightarrow \sqrt{\omega} x_{j}$ and $H \rightarrow \omega H$. It was shown by Calogero [2,3] that this Schrödinger operator has eigenfunctions of the form (2), with groundstate

$$
\Psi_{0}\left(x_{1}, \ldots, x_{N}\right)=\prod_{j=1}^{N} \mathrm{e}^{-(1 / 2) x_{j}^{2}} \prod_{j<k}\left(x_{k}-x_{j}\right)^{\kappa}
$$


corresponding to the eigenvalue

$$
E_{0}=N(1+\kappa(N-1)),
$$

and where the symmetric polynomials $P_{\lambda}$ are natural many-variable generalisations of the Hermite polynomials. This implies that these symmetric polynomials are eigenfunctions of the differential operator

$$
\begin{aligned}
\tilde{H}_{N} & :=\Psi_{0}^{-1}\left(H_{N}-E_{0}\right) \Psi_{0} \\
& =-\sum_{j=1}^{N} \frac{\partial^{2}}{\partial x_{j}^{2}}+2 \sum_{j=1}^{N} x_{j} \frac{\partial}{\partial x_{j}}-2 \kappa \sum_{j<k} \frac{1}{x_{j}-x_{k}}\left(\frac{\partial}{\partial x_{j}}-\frac{\partial}{\partial x_{k}}\right) .
\end{aligned}
$$

The idea is now to compute the action of this differential operator on the so-called monomial symmetric polynomials $m_{\lambda}$, for each partition $\lambda=\left(\lambda_{1}, \ldots, \lambda_{N}\right)$ defined by

$$
m_{\lambda}\left(x_{1}, \ldots, x_{N}\right)=\sum_{P} x_{1}^{\lambda_{P(1)}} \cdots x_{N}^{\lambda_{P(N)}}
$$

where the sum extends over all distinct permutations $P$ of the parts $\lambda_{j}$ of the partition $\lambda$. In the discussion below we will on occasion refer to monomials $m_{n}$ parametrised by integer vectors $n \in \mathbb{N}_{0}^{N}$ which are not partitions. Such a monomial is then defined by the equality $m_{n}=m_{p(n)}$, where $p(n)$ refers to the unique partition obtained by permuting the parts $n_{j}$ of $n$. Note that as $\lambda$ runs through all partitions of length at most $N$ the monomials $m_{\lambda}$ form a linear basis for the space of symmetric polynomials in $N$ variables. Using the fact that

$$
\left(-\frac{\partial}{\partial x}+2 x \frac{\partial}{\partial x}\right) x^{n}=2 n x^{n}-n(n-1) x^{n-2},
$$

as well as the identity

$$
\frac{1}{x-y}\left(\frac{\partial}{\partial x}-\frac{\partial}{\partial y}\right)\left(x^{n} y^{m}+y^{n} x^{m}\right)=(n-m) \sum_{k=1}^{n-m-1} x^{n-1-k} y^{m-1+k}-m\left(x^{n-1} y^{m-1}+y^{n-1} x^{m-1}\right),
$$

valid for all $x, y \in \mathbb{R}$ and $n, m \in \mathbb{N}_{0}$ such that $n \geq m$, it is straightforward to verify that

$$
\begin{aligned}
\tilde{H}_{N} m_{\lambda}= & 2|\lambda| m_{\lambda}-\sum_{j=1}^{N} \lambda_{j}\left(\lambda_{j}-1\right) m_{\lambda-2 e_{j}}-2 \kappa \sum_{j<k} \sum_{\nu=1}^{\left\lfloor\left(\lambda_{j}-\lambda_{k}\right) / 2\right\rfloor}\left(\lambda_{j}-\lambda_{k}\right) m_{\lambda-(\nu+1) e_{j}+(\nu-1) e_{k}} \\
& +2 \kappa \sum_{j<k} \lambda_{k} m_{\lambda-e_{j}-e_{k}},
\end{aligned}
$$

where $|\lambda|=\lambda+\cdots+\lambda_{N},\lfloor n / 2\rfloor$ denotes the integer part of $n / 2$, and $e_{j}$ are the natural basis elements in $\mathbb{Z}^{N}$ defined by $\left(e_{j}\right)_{k}=\delta_{j k}$. It is important to note at this point that the right hand side of this expression involves terms which in general are not parametrised by partitions, e.g., if $\lambda=(3,2,2)$ then $\lambda-2 e_{1}=(1,2,2)$ which is not a partition. However, since $m_{P(\lambda)}=m_{\lambda}$, for all permutations $P$ of $N$ objects, we can remedy this problem by collecting all terms corresponding to the same monomial $m_{\lambda}$. Once this is done we find that the action of the differential operator (7) on the monomials $m_{\lambda}$ is triangular, in the sense that if two partitions $\mu=\left(\mu_{1}, \ldots, \mu_{N}\right)$ and $\lambda=\left(\lambda_{1}, \ldots, \lambda_{N}\right)$ are ordered according to the partial ordering

$$
\mu \leq \lambda \Leftrightarrow \mu_{1}+\cdots+\mu_{j} \leq \lambda_{1}+\cdots+\lambda_{j}, \quad \forall j=1, \ldots, N
$$


then $\tilde{H} m_{\lambda}$ is a linear combination of $m_{\lambda}$ and monomials $m_{\mu}$ with $\mu<\lambda$ and $|\mu| \leq|\lambda|-2$, i.e.,

$$
\tilde{H}_{N} m_{\lambda}=2|\lambda| m_{\lambda}+\sum_{\mu} c_{\lambda \mu} m_{\mu}
$$

for some coefficients $c_{\lambda \mu}$, and where the sum is over partitions $\mu<\lambda$ such that $|\mu| \leq|\lambda|-2$. This means that when constructing the reduced eigenfunctions of the Calogero model we can restrict the differential operator (7) to a subspace of the symmetric polynomials spanned by monomials $m_{\mu}$, where $\mu \leq \lambda$ for some fixed partition $\lambda$. On this subspace the differential operator (7) can indeed be represented by a finite dimensional triangular matrix, with offdiagonal elements $c_{\lambda \mu}$, and where its diagonal elements $2|\lambda|$ give the eigenvalues for the reduced eigenfunctions of the Calogero model, which correspond to the eigenvectors of this matrix. There remains then to actually compute the matrix elements $c_{\lambda \mu}$, i.e., to collect all terms in (8) corresponding to the same monomial $m_{\mu}$. It seems however that this problem does not have a simple solution, which in turn implies that the reduced eigenfunctions of the Calogero model do not have a simple series representation in terms of monomial symmetric polynomials. The situation is similar for the other models discussed above (see e.g. [8]), and as far as we know also for other simple bases of the space of symmetric polynomials, such as elementary, complete homogeneous and power sum symmetric polynomials; see e.g. [17] for their definition.

\subsection{A sketch of our solution method}

To obtain our explicit analytic series representation for the reduced eigenfunctions of CalogeroSutherland type models with polynomial eigenfunctions we use a construction which differs from the one discussed above in two important aspects: first, we express them in terms of a particular set of symmetric polynomials $f_{n}, n \in \mathbb{Z}^{N}$, on which the action of the differential operator (4) is simpler than on the symmetric monomials; second, we avoid the problem of computing the matrix elements analogous to the $c_{\lambda \mu}$ in (9) by using an overcomplete set of these polynomials, parametrised not only by partitions but by a larger set of integer vectors in $\mathbb{Z}^{N}$. One could of course apply this latter change to the symmetric monomials and the discussion in the previous section. Note, however, that the expression (8) for the action of the differential operator (7) on the symmetric monomials is valid only for partitions, and that a formula valid for arbitrary integer vectors in $\mathbb{N}_{0}^{N}$ would be more involved.

To simplify notation we will here, and in the remainder of the paper, let $x=\left(x_{1}, \ldots, x_{N}\right)$ and $y=\left(y_{1}, \ldots, y_{N}\right)$ be two sets of independent variables. For an arbitrary integer vector $n \in \mathbb{Z}^{N}$ we will furthermore use the notation $x^{n}=x_{1}^{n_{1}} \cdots x_{N}^{n_{N}}$, and similarly for $y$. We now define the set of symmetric polynomials $f_{n}, n \in \mathbb{Z}^{N}$, through the expansion of their generating function

$$
\frac{\prod_{j<k}\left(1-\frac{y_{j}}{y_{k}}\right)^{\kappa}}{\prod_{j, k}\left(1-\frac{x_{j}}{y_{k}}\right)^{\kappa}}=\sum_{n \in \mathbb{Z}^{N}} f_{n}(x) y^{-n},
$$

valid for $\left|y_{N}\right|>\cdots>\left|y_{1}\right|>\max _{j}\left(\left|x_{j}\right|\right)$. Although the expansion unavoidably generates terms parametrised by integer vectors which are not partitions, we prove in Section 2.3 that a basis for the space of symmetric polynomials is formed by those $f_{n}$ which are parametrised by partitions alone. The reason that we use precisely these symmetric polynomials is that for each Schrödinger operator (1) there exists an identity

$$
H_{N}(x) F(x, y)=\left(H_{N}^{(-)}(y)+C_{N}\right) F(x, y),
$$


where $C_{N}$ is a constant, $H_{N}^{(-)}$is obtained from $H_{N}$ by a simple shift in its parameters (see [8]), and the function $F$ is given by

$$
F(x, y)=\Psi_{0}(x) \prod_{j=1}^{N} \psi_{0}^{(-)}\left(y_{j}\right) \frac{\prod_{j<k}\left(z\left(y_{k}\right)-z\left(y_{j}\right)\right)^{\kappa}}{\prod_{j, k}\left(z\left(y_{k}\right)-z\left(x_{j}\right)\right)^{\kappa}},
$$

where $\psi_{0}^{(-)}$is the groundstate of the one-body model obtained by setting $N=1$ in $H_{N}^{(-)}$. Note that if the groundstate factors are removed and the variables $z_{j}=z\left(x_{j}\right)$ and $w_{j}=z\left(y_{k}\right)$ are introduced we essentially recover the generating function for the symmetric polynomials $f_{n}$. This relation will later enable us to obtain the action of the differential operator (4) on the $f_{n}$ in a straightforward manner. As is then shown, this action is simple enough to be inverted explicitly, thus yielding our explicit analytic series representation for the reduced eigenfunctions of Calogero-Sutherland type models with polynomial eigenfunctions.

In the literature there exist various other approaches to the construction of these reduced eigenfunctions. In a recent paper Lassalle and Schlosser [16] obtained two explicit analytic series expansions for the Jack polynomials, the reduced eigenfunctions of the Sutherland model, by inverting their so called Pieri formula. For very particular partitions or a low number of variables explicit analytic expansions have also been obtained by other methods; see e.g. [17]. In addition, various representations of a combinatorial nature are known for the Jack, as well as certain other related many-variable polynomials $[5,9,17,27]$. We also mention the recent separation-of-variables approach to the Sutherland model due to Kuznetsov, Mangazeev and Sklyanin [10], which also relies on the identity (11). This list of previous results reflects only those which we have found to be most closely related to ours. For a more comprehensive discussion we refer to [8].

\subsection{An outline for the remainder of the paper}

We continue in Section 2 to give a more detailed account of our solution method by applying it to the particular case of the Calogero model. In Section 3 we then discuss generalisations of this result to other Calogero-Sutherland type models with polynomial eigenfunctions and also to the 'deformed' Calogero-Sutherland type models recently introduced and studied by Chalykh, Feigin, Sergeev and Veselov; see [4, 21, 22] and references therein.

\section{A first example: eigenfunctions of the Calogero model}

In this section we provide a detailed account of our solution method by applying it to the Calogero model, defined by the Schrödinger operator (5). Apart from the proof of completeness these results were all obtained in [7].

We begin by formulating our main result: an explicit analytic series representation for the reduced eigenfunctions of the Calogero model in terms of the symmetric polynomials $f_{n}$. In doing so we make use of a few notational conventions which we now introduce. In contrast to the introduction we will here use the following partial ordering ordering of integer vectors $m, n \in \mathbb{Z}^{N}$ :

$$
m \preceq n \Leftrightarrow m_{j}+\cdots+m_{N} \leq n_{j}+\cdots+n_{N}, \quad \forall j=1, \ldots, N .
$$

To simplify certain formulae we associate to each $n \in \mathbb{Z}^{N}$ the shifted integer vector

$$
n^{+}=\left(n_{1}^{+}, \ldots, n_{N}^{+}\right), \quad n_{j}^{+}=n_{j}+\kappa(N+1-j) .
$$


For each integer vector $n \in \mathbb{Z}^{N}$ we define the Kronecker delta

$$
\delta_{n}(m)=\prod_{j=1}^{N} \delta_{n_{j} m_{j}} .
$$

We also recall the notation $e_{j}$ for the standard basis in $\mathbb{Z}^{N}$, i.e., $\left(e_{j}\right)_{k}=\delta_{j k}$. We are now ready to state the main result of this section.

Theorem 1. For an arbitrary integer vector $n \in \mathbb{Z}^{N}$ let

$$
P_{n}=f_{n}+\sum_{m} u_{n}(m) f_{m}
$$

where the sum is over integer vectors $m \in \mathbb{Z}^{N}$ such that

$$
m \prec n \text { and }|m| \leq|n|-2 \text {, }
$$

and the coefficients

$$
u_{n}(m)=\sum_{s=1}^{\infty} \frac{1}{4^{s} s !} \sum_{j_{1} \leq k_{1}} \cdots \sum_{j_{s} \leq k_{s}} \sum_{\nu_{1}, \ldots, \nu_{s}=0}^{\infty} \delta_{n}\left(m+\sum_{r=1}^{s} E_{j_{r} k_{r}}^{\nu_{r}}\right) \prod_{r=1}^{s} g_{j_{r} k_{r}}\left(\nu_{r} ; n-\sum_{\ell=1}^{r} E_{j_{\ell} k_{\ell}}^{\nu_{\ell}}\right),
$$

where we use the shorthand notation

$$
g_{j k}(\nu ; m)=2 \kappa(\kappa-1) \nu\left(1-\delta_{j k}\right)-m_{j}^{+}\left(m_{j}^{+}+1\right) \delta_{\nu 0} \delta_{j k}
$$

and

$$
E_{j k}^{\nu}=(1-\nu) e_{j}+(1+\nu) e_{k}
$$

Then $P_{n}$ is a reduced eigenfunction of the Schrödinger operator (5) corresponding to the eigenvalue

$$
E_{n}=2|n|+E_{0}, \quad E_{0}=N(1+\kappa(N-1)) .
$$

Moreover, as $\lambda$ runs through all partitions of length at most $N$ the $P_{\lambda}$ form a basis for the space of symmetric polynomials in $N$ variables.

Remark 1. It is important to note that the series defining the coefficients $u_{n}(m)$ terminate after a finite number of terms, and thus are well-defined. This is a direct consequence of the definition of the Kronecker-delta $\delta_{n}(m)$ and the fact that the equations

$$
n-m=\sum_{r=1}^{s} E_{j_{r} k_{r}}^{\nu_{r}}
$$

only have a finite number of solutions $\nu=\left(\nu_{1}, \ldots, \nu_{s}\right)$ for fixed $n, m \in \mathbb{Z}^{N}$.

To prove the theorem we proceed in three steps: we begin by deriving the identity (11) for the Schrödinger operator (5); we then prove the first part of the theorem, that the functions $P_{n}$ are reduced eigenfunctions of the Schrödinger operator (5); and finally, we prove that a basis for its eigenspace is given by those eigenfunctions which are parametrised by a partition of length at most $N$. 


\subsection{The identity and a model with different masses}

Rather than proving the identity (11) for the Schrödinger operator (5) by a direct computation we obtain it here as a consequence of a more general result which has the interpretation of providing the exact groundstate of a generalisation of the Calogero model where the particles are allowed to have different masses. We will, however, not stress this interpretation but rather use the result to derive various other identities, of which (11) is the one of main interest for the discussion which follows.

Proposition 1. For a given set of real non-zero parameters $m=\left(m_{1}, \ldots, m_{\mathcal{N}}\right)$ and variables $X=\left(X_{1}, \ldots, X_{\mathcal{N}}\right)$ let

$$
\mathcal{H}=-\sum_{j=1}^{\mathcal{N}} \frac{1}{m_{j}} \frac{\partial^{2}}{\partial X_{j}^{2}}+\sum_{j=1}^{\mathcal{N}} m_{j} X_{j}^{2}+\kappa \sum_{j<k}\left(\kappa m_{j} m_{k}-1\right)\left(m_{j}+m_{k}\right) \frac{1}{\left(X_{j}-X_{k}\right)^{2}}
$$

and let

$$
\Phi_{0}\left(X_{1}, \ldots, X_{\mathcal{N}}\right)=\prod_{j=1}^{\mathcal{N}} \psi_{0, m_{j}}\left(X_{j}\right) \prod_{j<k}\left(X_{k}-X_{j}\right)^{\kappa m_{j} m_{k}}, \quad \psi_{0, m_{j}}\left(X_{j}\right)=\mathrm{e}^{-m_{j} X_{j}^{2} / 2} .
$$

We then have that

$$
\mathcal{H} \Phi_{0}=\mathcal{E}_{0} \Phi_{0}
$$

with the constant

$$
\mathcal{E}_{0}=\kappa\left(\sum_{j=1}^{\mathcal{N}} m_{j}\right)^{2}+\sum_{j=1}^{\mathcal{N}}\left(1-\kappa m_{j}^{2}\right)
$$

Moreover, if all $m_{j}$ are positive and $\Phi_{0}$ is square integrable then $\mathcal{H}$ defines a self-adjoint operator bounded from below by $\mathcal{E}_{0}$ and with groundstate $\Phi_{0}$.

Proof. We prove the statement by establishing that the differential operator (14) is factorisable according to

$$
\mathcal{H}=\sum_{j=1}^{\mathcal{N}} \frac{1}{m_{j}} Q_{j}^{+} Q_{j}^{-}+\mathcal{E}_{0}
$$

with

$$
Q_{j}^{ \pm}= \pm \partial_{X_{j}}+\mathcal{V}_{j}, \quad \mathcal{V}_{j}=\Phi_{0}^{-1} \partial_{X_{j}} \Phi_{0}
$$

Note that $Q_{j}^{+}$is the formal adjoint of $Q_{j}^{-}$. The identity (15) then follows from the fact that $Q_{j}^{-} \Phi_{0}=0$ for all $j$. If all $m_{j}$ are positive then this factorisation shows that $\mathcal{H}$ defines a unique self-adjoint operator via the Friedrichs extension which is bounded from below by $\mathcal{E}_{0}$ (see e.g. Theorem X.23 in [20]) and with $\Phi_{0}$ as ground state.

Observing that

$$
\mathcal{V}_{j}\left(X_{1}, \ldots, X_{\mathcal{N}}\right)=-m_{j} X_{j}+\kappa \sum_{k \neq j} m_{j} m_{k} \frac{1}{X_{j}-X_{k}}
$$


it is straightforward to deduce that

$$
\sum_{j=1}^{\mathcal{N}} \frac{1}{m_{j}} Q_{j}^{+} Q_{j}^{-}=\mathcal{H}-\mathcal{R}
$$

with remainder term

$$
\mathcal{R}=2 \kappa \sum_{k \neq j} m_{j} m_{k} \frac{X_{j}}{X_{j}-X_{k}}+\kappa^{2} \sum_{\substack{k, l \neq j \\ l \neq k}} \frac{m_{j} m_{k} m_{l}}{\left(X_{k}-X_{j}\right)\left(X_{j}-X_{l}\right)}+\mathcal{N} .
$$

Upon symmetrising the double sum and using the identity

$$
\frac{1}{\left(X_{k}-X_{j}\right)\left(X_{j}-X_{l}\right)}+\frac{1}{\left(X_{l}-X_{k}\right)\left(X_{k}-X_{j}\right)}+\frac{1}{\left(X_{j}-X_{l}\right)\left(X_{l}-X_{k}\right)}=0
$$

it is readily verified that

$$
\mathcal{R}=\kappa \sum_{k \neq j} m_{j} m_{k}+\mathcal{N}=\kappa\left(\sum_{j=1}^{\mathcal{N}} m_{j}\right)^{2}+\sum_{j=1}^{\mathcal{N}}\left(1-\kappa m_{j}^{2}\right)=\mathcal{E}_{0} .
$$

We note that by setting all $m_{j}=1$ we obtain as a direct consequence of the proposition that (6) indeed is the groundstate of the Calogero model. On the other hand, setting $\mathcal{N}=2 N$, $m_{j}=1$ and $m_{N+j}=-1$ for $j=1, \ldots, N$ we see that $\mathcal{H}$ splits into a difference of two Schrödinger operators (5) and that we obtain the corresponding identity (11) with $H_{N}^{(-)}=H_{N}$.

Corollary 1. With

$$
F(x, y)=\Psi_{0}(x) \prod_{j=1}^{N} \psi_{0,-1}\left(y_{j}\right) \frac{\prod_{j<k}\left(y_{k}-y_{j}\right)^{\kappa}}{\prod_{j, k}\left(y_{k}-x_{j}\right)^{\kappa}}
$$

we have that

$$
H_{N}(x) F(x, y)=\left(H_{N}(y)+C_{N}\right) F(x, y),
$$

where the constant

$$
C_{N}=2(1-\kappa) N
$$

It is interesting to observe that Proposition 1 implies a number of additional identities. We can for example choose to take different number of variables $x_{j}$ and $y_{k}$. This leads to an identity involving two Schrödinger operators $H_{N}$ and $H_{M}$ with different number of variables $N$ and $M$. We may also set some of the parameters $m_{j}$ to either $1 / \kappa$ or $-1 / \kappa$ while still preserving the property that $\mathcal{H}$ splits into a difference of two differential operators, which in this case will define so-called 'deformed' Calogero-Sutherland type models; see Section 3.2. These additional identities are further discussed in [8].

\subsection{Construction of reduced eigenfunctions}

We proceed to prove the first part of the statement in Theorem 1, that the symmetric polynomials $P_{n}$, defined by (12), are reduced eigenfunctions of the Schrödinger operator (5). We begin by computing the action of the differential operator $(7)$ on the symmetric polynomials $f_{n}$. 
Lemma 1. For each $n \in \mathbb{Z}^{N}$ we have that

$$
\tilde{H}_{N} f_{n}=\tilde{E}_{n} f_{n}-\sum_{j=1}^{N}\left(n_{j}^{+}-1\right)\left(n_{j}^{+}-2\right) f_{n-2 e_{j}}+2 \kappa(\kappa-1) \sum_{j<k} \sum_{\nu=1}^{\infty} \nu f_{n-(1-\nu) e_{j}-(1+\nu) e_{k}}
$$

with

$$
\tilde{E}_{n}=E_{n}-E_{0}=2|n| .
$$

Proof. We first note that the function $F$ in Corollary 1 and the generating function for the symmetric polynomials $f_{n}$ are related as follows:

$$
F(x, y)=\Psi_{0}(x)\left(\prod_{j=1}^{N} \psi_{0,-1}\left(y_{j}\right) y_{j}^{-\kappa(N+1-j)}\right) \frac{\prod_{j<k}\left(1-\frac{y_{j}}{y_{k}}\right)^{\kappa}}{\prod_{j, k}\left(1-\frac{x_{j}}{y_{k}}\right)^{\kappa}}
$$

with $\Psi_{0}$ the groundstate (6) of the Schrödinger operator (5). The identity (16) in Corollary 1 together with definitions $(7)$ and (10), of respectively the differential operator $\tilde{H}_{N}$ and the symmetric polynomials $f_{n}$, therefore imply that

$$
\sum_{n \in \mathbb{Z}^{N}}\left(\tilde{H}_{N} f_{n}(x)\right) y^{-n^{+}}=\sum_{n \in \mathbb{Z}^{N}} f_{n}(x)\left(\bar{H}_{N}+C_{N}-E_{0}\right) y^{-n^{+}}
$$

where

$$
\begin{aligned}
\bar{H}_{N} & =\frac{1}{\prod_{j=1}^{N} \psi_{0,-1}\left(y_{j}\right)} H_{N} \prod_{j=1}^{N} \psi_{0,-1}\left(y_{j}\right) \\
& =-\sum_{j=1}^{N} \frac{\partial^{2}}{\partial y_{j}^{2}}-\sum_{j=1}^{N}\left(2 y_{j} \frac{\partial}{\partial y_{j}}+1\right)+2 \kappa(\kappa-1) \sum_{j<k} \frac{1}{\left(y_{j}-y_{k}\right)^{2}} .
\end{aligned}
$$

We now expand the interaction term in a geometric series

$$
\frac{1}{\left(y_{j}-y_{k}\right)^{2}}=\sum_{\nu=1}^{\infty} \nu \frac{y_{j}^{\nu-1}}{y_{k}^{\nu+1}}
$$

which in the region $\left|y_{N}\right|>\left|y_{N-1}\right|>\cdots>\left|y_{1}\right|$ is valid for all $j<k$. It is now straightforward to compute the right hand side of (19), and by comparing coefficients of $y^{-n^{+}}$on both sides of the resulting equation we obtain (17) with

$$
\tilde{E}_{n}=\sum_{j=1}^{N}\left(2 n_{j}^{+}-1\right)+C_{N}-E_{0} .
$$

As a simple computation shows, this indeed coincides with (18), and the statement is thereby proved.

Remark 2. At this point it is interesting to compare the action of the differential operator (7) on the monomials $m_{\lambda}$, given by (8), and on the polynomials $f_{n}$, as just obtained. We note, in particular, that the simpler structure of the latter arise from the fact that it is essentially equivalent to the action of the differential operator $\bar{H}_{N}$, defined by (20), on the powers $y^{-n^{+}}$ and the fact that the 'interaction' terms of this operator does not contain any derivatives, in contrast to the differential operator (7). 
It is clear from (17) that the action of the differential operator (7) on the symmetric polynomials $f_{n}$ has a triangular structure, in the sense that $\tilde{H} f_{n}$ is a linear combination of $f_{n}$ and symmetric polynomials $f_{m}$ with $m \prec n$ and $|m| \leq|n|-2$. This suggests that to each $n \in \mathbb{Z}^{N}$ corresponds a reduced eigenfunction $P_{n}$ of the form (12) with eigenvalue $\tilde{E}_{n}$. Inserting this ansatz into (17) and introducing $u_{n}(n)=1$ we obtain

$$
\begin{aligned}
\tilde{H}_{N} P_{n}= & \tilde{E}_{n} f_{n}+\sum_{m}\left(\tilde{E}_{m} u_{n}(m)-\sum_{j=1}^{N}\left(m_{j}^{+}+1\right) m_{j}^{+} u_{n}\left(m+2 e_{j}\right)\right. \\
& \left.+2 \kappa(\kappa-1) \sum_{j<k} \sum_{\nu=1}^{\infty} \nu u_{n}\left(m+(1-\nu) e_{j}+(1+\nu) e_{k}\right)\right) f_{m},
\end{aligned}
$$

where the sum is over integer vectors $m \in \mathbb{Z}^{N}$ such that $m \prec n$ and $|m| \leq|n|-2$. We therefore conclude that the validity of the Schrödinger equation $H_{N} \Psi_{n}=E_{n} \Psi_{n}$ follows from the recursion relation

$$
2(|n|-|m|) u_{n}(m)=\sum_{j \leq k} \sum_{\nu=0}^{\infty} g_{j k}(\nu ; m) u_{n}\left(m+E_{j k}^{\nu}\right),
$$

with the coefficients $g_{j k}(\nu ; m)$ defined by $(13)$ and where we used the fact that $\tilde{E}_{n}-\tilde{E}_{m}=$ $2(|n|-|m|)$. We now proceed to solve this recursion relation. Suppressing the argument $m$ we rewrite it in the form

$$
u_{n}=\delta_{n}+R u_{n},
$$

where the operator $R$ is defined by

$$
\left(R u_{n}\right)(m)=\frac{1}{2(|n|-|m|)} \sum_{j \leq k} \sum_{\nu=0}^{\infty} g_{j k}(\nu ; m) u_{n}\left(m+E_{j k}^{\nu}\right) .
$$

Observe that this expression is well-defined since $|n|-|m| \neq 0$ for all applicable $m$ and the sum truncates after a finite number of terms: $u_{n}(m)$ is by definition non-zero only if $m \preceq n$. The solution of this latter equation is therefore

$$
u_{n}=(1-R)^{-1} \delta_{n}=\sum_{s=0}^{\infty} R^{s} \delta_{n}
$$

where the expansion into a geometric series is well-defined since it only contains a finite number of non-zero terms, as will become apparent below. Using the definition of the operator $R$ as well as the defining properties of the Kronecker delta $\delta_{n}$ we deduce that

$$
\begin{aligned}
\left(R^{s} \delta_{n}\right)(m)= & \sum_{j_{s} \leq k_{s}} \sum_{\nu_{s}=0}^{\infty} \frac{g_{j_{s} k_{s}}\left(\nu_{s} ; m\right)}{2(|n|-|m|)} \sum_{j_{s-1} \leq k_{s}-1} \sum_{\nu_{s-1}=0}^{\infty} \frac{g_{j_{s-1} k_{s-1}}\left(\nu_{s-1} ; m+E_{j_{s} k_{s}}^{\nu_{s}}\right)}{2\left(|n|-\left|m+E_{j_{s} k_{s}}^{\nu_{s}}\right|\right)} \times \cdots \\
& \times \sum_{j_{1} \leq k_{1}} \sum_{\nu_{1}=0}^{\infty} \frac{g_{j_{1} k_{1}}\left(\nu_{1} ; m+\sum_{\ell=2}^{s} E_{j_{\ell} k_{\ell}}^{\nu_{\ell}}\right)}{2\left(|n|-\left|m+\sum_{\ell=2}^{s} E_{j_{\ell} k_{\ell} \mid}^{\nu_{\ell}}\right|\right)} \delta_{n}\left(m+\sum_{r=1}^{s} E_{j_{r} k_{r}}^{\nu_{r}}\right) \\
= & \sum_{j_{1} \leq k_{1}} \cdots \sum_{j_{s} \leq k_{s}} \sum_{\nu_{1}, \ldots, \nu_{s}=0}^{\infty} \delta_{n}\left(m+\sum_{r=1}^{s} E_{j_{r} k_{r}}^{\nu_{r}}\right) \prod_{r=1}^{s} \frac{g_{j_{r} k_{r}}\left(\nu_{r} ; n-\sum_{\ell=1}^{r} E_{j_{\ell} k_{\ell}}^{\nu_{\ell}}\right)}{2\left(|n|-\left|n-\sum_{\ell=1}^{r} E_{j_{\ell} k_{\ell}}^{\nu_{\ell}}\right|\right)} .
\end{aligned}
$$


By finally observing that

$$
2\left(|n|-\left|n-\sum_{\ell=1}^{r} E_{j_{\ell} k_{\ell}}^{\nu_{\ell}}\right|\right)=4 r
$$

we obtain our explicit analytic series representation (12) for the reduced eigenfunctions of the Calogero model.

\subsection{Completeness of the reduced eigenfunctions}

There remains only to prove that the reduced eigenfunctions just obtained provide a basis for the space of symmetric polynomials, i.e., that they span the eigenspace of the differential operator (7). We obtain this last part of Theorem 1 by exploiting the relation between the symmetric polynomials $f_{n}$ and the so-called 'modified complete' symmetric polynomials $g_{\lambda}$, defined through the expansion of their generating function

$$
\frac{1}{\prod_{j, k}\left(1-\frac{x_{j}}{y_{k}}\right)^{\kappa}}=\sum_{\lambda} g_{\lambda}(x) m_{\lambda}\left(y^{-1}\right)
$$

valid for $\min _{k}\left|y_{k}\right|>\max _{j}\left(\left|x_{j}\right|\right)$, and where the summation extends over all partitions of length at most $N$. It is well known that the $g_{\lambda}$ are homogeneous symmetric polynomials of degree $|\lambda|$, and also that as $\lambda$ runs through all partitions of length at most $N$ they form a basis for the space of symmetric polynomials in $N$ variables; see e.g. Section VI.10 in [17]. We mention that these first properties can be directly inferred from their generating function, whereas the fact that they span the space of symmetric polynomials is a consequence of the equivalence between the expansion by which they are defined and the fact that they are dual to the to the monomial symmetric polynomials $m_{\lambda}$ in a particular inner product; see e.g. Statement 10.4 in [17].

By comparing the generating functions for the $f_{n}$ and the $g_{\lambda}$ we find that

$$
\sum_{n} f_{n}(x) y^{-n}=\prod_{j<k}\left(1-\frac{y_{j}}{y_{k}}\right)^{\kappa} \sum_{\lambda} g_{\lambda}(x) m_{\lambda}\left(y^{-1}\right) .
$$

Assuming $\left|y_{N}\right|>\cdots>\left|y_{1}\right|$ and expanding each term in the product in a power series we rewrite the right hand side as follows:

$$
\sum_{n \in \mathbb{N}_{0}^{N}} g_{p(n)}(x) \prod_{j<k} \sum_{p_{j k}=0}^{\infty}(-1)^{p_{j k}}\left(\begin{array}{c}
\kappa \\
p_{j k}
\end{array}\right) y^{-n+\sum_{j<k} p_{j k}\left(e_{j}-e_{k}\right)},
$$

where we have taken $p(n)$ to denote the unique partition obtained by reordering the parts $n_{j}$ of $n$. This means that

$$
f_{n}=\prod_{j<k} \sum_{p_{j k}=0}^{\infty}(-1)^{p_{j k}}\left(\begin{array}{c}
\kappa \\
p_{j k}
\end{array}\right) g_{p\left(n+\sum_{j<k} p_{j k}\left(e_{j}-e_{k}\right)\right)}
$$

Many of the properties of the 'modified complete' symmetric polynomials $g_{\lambda}$ for this reason carry over to the $f_{n}$. In particular, observing that

$$
\left|n+\sum_{j<k} p_{j k}\left(e_{j}-e_{k}\right)\right|=|n|
$$


for all integers $p_{j k}$ we conclude that they are homogeneous symmetric polynomials of degree $n$. We also see that they are non-zero only if $n \succeq 0$. Now suppose $\lambda$ is a partition. It is then clear that

$$
m:=\lambda+\sum_{j<k} p_{j k}\left(e_{j}-e_{k}\right) \preceq \lambda
$$

and furthermore that also $p(m) \preceq \lambda$ : we obtain the partition $p(m)$ from $m$ by some permutation of its parts $m_{j}$, and since by definition $\mu_{1} \geq \cdots \geq \mu_{N}$ for any partition $\mu=\left(\mu_{1}, \ldots, \mu_{N}\right)$ we have that $p(m) \preceq m \preceq \lambda$. We therefore conclude that

$$
f_{\lambda}=g_{\lambda}+\sum_{\mu} M_{\lambda \mu} g_{\mu}
$$

for some coefficients $M_{\lambda \mu}$, and where the sum is over partitions $\mu \prec \lambda$. As indicated in this expression we let $M=\left(M_{\lambda \mu}\right)$ denote the transition matrix, defined by the equality $f_{\lambda}=$ $\sum_{\mu} M_{\lambda \mu} g_{\mu}$, from the $f_{\lambda}$ to the $g_{\mu}$. Given a partition $\lambda$ it follows from (22) that it can be consistently restricted to the partitions $\mu$ such that $\mu \preceq \lambda$. With rows and columns ordered in descending order this restricted transition matrix is upper triangular with 1's on the diagonal. Hence, it can be inverted. Since the inverse of an upper triangular matrix is upper triangular we obtain that

$$
g_{\lambda}=f_{\lambda}+\sum_{\mu}\left(M^{-1}\right)_{\lambda \mu} f_{\mu}
$$

where the sum is over partitions $\mu \prec \lambda$. We have thereby proved the following:

Proposition 2. The functions $f_{n}$ are non-zero only if $n \succeq 0$. In that case, $f_{n}$ is a homogeneous symmetric polynomial of degree $|n|$. Moreover, as $\lambda$ runs through all partitions of length at most $N$ the $f_{\lambda}$ form a basis for the space of symmetric polynomials in $N$ variables.

The same line of reasoning can now be applied to the reduced eigenfunctions

$$
P_{\lambda}=f_{\lambda}+\sum_{m} u_{\lambda}(m) f_{m}
$$

which are parametrised by partitions $\lambda$. Recall that the sum is over integer vectors $m \prec \lambda$. Using formula (21) and following the subsequent discussion we find that

$$
P_{\lambda}=g_{\lambda}+\sum_{\mu} b_{\lambda \mu} g_{\mu}
$$

for some coefficients $b_{\lambda \mu}$, and where the sum now extends only over partitions $\mu \prec \lambda$. Applying the arguments leading up to Proposition 2 we conclude that this expression can be inverted to yield each $g_{\lambda}$ as a linear combination of the $P_{\mu}$ with $\mu \preceq \lambda$. Hence, as $\lambda$ runs through all partitions of length at most $N$ the $P_{\lambda}$ form a basis for the space of symmetric polynomials in $N$ variables. This concludes the proof of Theorem 1.

\section{Generalisations to other models}

In this section we indicate how the results on the Calogero model obtained in the previous section can be generalised to similar models with polynomial eigenfunctions, including not only models of Calogero-Sutherland type but also the 'deformed' Calogero-Sutherland models introduced and studied by Chalykh et.al.; see [4, 21, 22] and references therein. A detailed account of these results is in preparation [8]. 


\subsection{Calogero-Sutherland models with polynomial eigenfunctions}

When the number of particles are set to one in the Calogero model it reduces to the very well known harmonic oscillator, which features eigenfunctions given by the classical Hermite polynomials. This is only one special case of the following well known and more general statement: to each complete sequence of polynomials $\left\{p_{n}: n \in \mathbb{N}_{0}\right\}$, obeying a second order ordinary differential equation, there is a corresponding Schrödinger operator

$$
h=-\frac{\partial^{2}}{\partial x^{2}}+V(x)
$$

with a particular potential function $V$ such that its eigenfunctions are of the form

$$
\psi_{n}(x)=\psi_{0}(x) p_{n}(z(x))
$$

for some functions $\psi_{0}$ and $z$. This can be verified by first observing that such a set of polynomials are eigenfunctions of a differential operator

$$
\tilde{h}=\alpha(z) \frac{\partial^{2}}{\partial z^{2}}+\beta(z) \frac{\partial}{\partial z},
$$

where

$$
\alpha(z)=\alpha_{2} z^{2}+\alpha_{1} z+\alpha_{0} \quad \text { and } \quad \beta(z)=\beta_{1} z+\beta_{0}
$$

for some coefficients $\alpha_{j}$ and $\beta_{j}$. Now introducing the variable $x=x(z)$ as a solution of the differential equation

$$
x^{\prime}(z)=\frac{1}{\sqrt{\alpha(z)}}
$$

and defining the function $\psi_{0}$ by

$$
\psi_{0}(x)=\mathrm{e}^{-w(z(x))}, \quad w^{\prime}=\frac{\alpha^{\prime}-2 \beta}{4 \alpha},
$$

it is straightforward to verify that a Schrödinger operator $h$ of the form (23) is obtained by conjugation of $\tilde{h}$ by the function $\psi_{0}$ and changing the independent variable to $x$, or to be more precise,

$$
h=-\psi_{0} \tilde{h} \psi_{0}^{-1}=-\frac{\partial^{2}}{\partial x^{2}}+V(x),
$$

with potential function

$$
V(x)=v(z(x)), \quad v=\frac{\left(2 \beta-\alpha^{\prime}\right)\left(2 \beta-3 \alpha^{\prime}\right)}{16 \alpha}+\frac{1}{4} \alpha^{\prime \prime}-\frac{1}{2} \beta^{\prime} .
$$

To illustrate this general discussion we have listed the particular values of the coefficients $\alpha_{j}$ and $\beta_{j}$, as well as associated functions $\psi_{0}$ and $z$, which correspond to the classical orthogonal polynomials (of Hermite, Laguerre and Jacobi) and the generalised Bessel polynomials in Table 1. In fact, we can by simple translations and rescalings always reduce to one of these four cases.

We mention that transformations of differential equations of the type described above are frequently used in the theory of ordinary differential equations of second order; see e.g. Section 1.8 in Szegö's classical book [25] on orthogonal polynomials. It is interesting to note that this 


\begin{tabular}{|c|c|c|c|c|}
\hline$p_{n}(z)$ & $\alpha(z)$ & $\beta(z)$ & $\psi_{0}(x)$ & $z(x)$ \\
\hline $\begin{array}{c}H_{n}(z) \\
(\text { Hermite }) \\
L_{n}^{(a)}\end{array}$ & 1 & $-2 z$ & $\mathrm{e}^{-x^{2} / 2}$ & $x$ \\
$\left(\begin{array}{c}\text { Laguerre }) \\
P_{n}^{(a, b)}(z) \\
(\text { Jacobi) } \\
y_{n}(z ; 1-2 a, 2 b) \\
(\text { gen. Bessel })\end{array}\right.$ & $1-z^{2}$ & $b-a-(a+b+2) z$ & $\sin ^{a+1 / 2}\left(\frac{x}{2}\right) \cos ^{b+1 / 2}\left(\frac{x}{2}\right)$ & $\cos x$ \\
\hline
\end{tabular}

Table 1. The particular values of coefficients $\alpha_{j}$ and $\beta_{j}$, as well as associated functions $\psi_{0}$ and $z$, corresponding to the classical orthogonal polynomials (of Hermite, Laguerre and Jacobi) and the generalised Bessel polynomials.

transformation has a simple and direct generalisation to many variables. Setting the interaction potential

$$
W(x, y)=\frac{\alpha(z(x))+\alpha(z(y))}{(z(x)-z(y))^{2}}
$$

and keeping the potential function $V$ as given above one verifies that the Schrödinger operator (1) after a conjugation by the function $\Psi_{0}$, as defined in (3), and a change of independent variables from the $x_{i}$ to the $z_{i}$, as defined above, is transformed into the differential operator

$$
\begin{aligned}
\tilde{H}_{N} & =-\Psi_{0}^{-1}\left(H-E_{0}\right) \Psi_{0} \\
& =\sum_{j=1}^{N} \alpha\left(z_{j}\right) \frac{\partial^{2}}{\partial z_{j}^{2}}+\sum_{j=1}^{N} \beta\left(z_{j}\right) \frac{\partial}{\partial z_{j}}+2 \kappa \sum_{j<k} \frac{1}{z_{j}-z_{k}}\left(\alpha\left(z_{j}\right) \frac{\partial}{\partial z_{j}}-\alpha\left(z_{k}\right) \frac{\partial}{\partial z_{k}}\right) .
\end{aligned}
$$

Following the discussion in Section 1.1 it is straightforward to verify that the action of this differential operator on the monomial symmetric polynomials is triangular in the very same ordering as in the case of the Calogero model. This means that the Schrödinger operator (1) for these choices of potential functions $V$ and $W$, up to degeneracies in its spectrum, has a complete set of reduced eigenfunctions given by symmetric polynomials. We mention that this unifying point of view on Calogero-Sutherland type models with polynomial eigenfunctions seems to have been little used in the literature, with the notable exception of Gomez-Ullate, González-López and Rodriguez [6] who, among other things, used this point of view to obtain the spectrum of all these models.

In [8] we show that our construction of an explicit series representation for the reduced eigenfunctions, presented in the previous section for the Calogero model, goes through virtually unchanged for all these models. In particular, we generalise Theorem 1 to the following:

Theorem 2. For $n \in \mathbb{Z}^{N}$, the reduced eigenfunctions of the Schrödinger operator (1) are formally given by

$$
P_{n}=f_{n}+\sum_{m} u_{n}(m) f_{m}
$$

where the sum is over integer vectors $m \in \mathbb{Z}^{N}$ such that

$$
m \prec n \text { and }|m| \leq|n|+\operatorname{deg}(\alpha)-2,
$$


and the coefficients

$$
u_{n}(m)=\sum_{l=1}^{\infty} \sum_{j_{1} \leq k_{1}} \cdots \sum_{j_{l} \leq k_{l}} \sum_{p_{1}, \ldots, p_{l}=0}^{2} \sum_{\nu_{1}, \ldots, \nu_{l}=1}^{\infty} \delta_{n}\left(m+\sum_{t=1}^{l} E_{j_{t} k_{t}}^{p_{t} \nu_{t}}\right) \prod_{r=1}^{l} \frac{g_{j_{r} k_{r}}\left(p_{r}, \nu_{r} ; n-\sum_{q=r}^{l} E_{j_{q} k_{q}}^{p_{q} \nu_{q}}\right)}{b_{n}\left(n-\sum_{q=r}^{l} E_{j_{q} k_{q}}^{p_{q} \nu_{q}}\right)}
$$

where we use the shorthand notation

$$
\begin{aligned}
b_{n}(m)=\tilde{E}_{n}- & \tilde{E}_{m}, \quad \tilde{E}_{n}=-\sum_{j=1}^{N}\left(\alpha_{2} n_{j}\left(n_{j}-1\right)+\left(\beta_{1}+2 \kappa(N-j)\right) n_{j}\right), \\
g_{j k}(p, \nu ; m)= & \left(1-\delta_{j k}\right) \kappa(\kappa-1) \alpha_{p}(2 \nu-p) \\
& -\delta_{j k} \delta_{\nu 1} m_{j}^{+}\left(\delta_{p 0} \alpha_{0}\left(m_{j}^{+}+1\right)+\delta_{p 1}\left(\alpha_{1}\left(m_{j}^{+}+\kappa+1\right)-\beta_{0}\right)\right)
\end{aligned}
$$

and

$$
E_{j k}^{p \nu}=(1-\nu) e_{j}+(1-p+\nu) e_{k}
$$

If $b_{n}(m) \neq 0$ for all integer vectors $m \in \mathbb{Z}^{N}$ such that $m \prec n$ and $|m| \leq|n|+\operatorname{deg}(\alpha)-2$ then $P_{n}$ is a well defined symmetric polynomial. Moreover, if this is the case for all integer vectors $n \in \mathbb{Z}^{N}$ such that $n=\lambda$ for some partition $\lambda$ of length at most $N$ then the corresponding $P_{\lambda}$ form a linear basis for the space of symmetric polynomials in $N$ variables.

Remark 3. It is important to note that the condition $b_{n}(m) \neq 0, m \prec n$ and $|m| \leq|n|+$ $\operatorname{deg}(\alpha)-2$, is essential in order for the coefficients $u_{n}(m)$ to be well defined. For generic choices of the parameter $\kappa$ and the polynomials $\alpha$ and $\beta$ it is satisfied for all $n \in \mathbb{Z}^{N}$; see [8] for a further discussion of this point.

Remark 4. At this point it is interesting to enquire whether our basis for the reduced eigenfunctions of the Schrödinger operator (1), as stated in Theorem 2, in applicable cases stand in a simple relation to the generalised hypergeometric polynomials of Lassalle $[13,14,15]$ and MacDonald [18], defined by expansions in Jack polynomials. In the case $\alpha=-z^{2}$ and $\beta=-z$, corresponding to the Jack polynomials themselves, one can show that they in fact coincide and it seems natural to expect this to be true also for the generalised Jacobi polynomials. Since the generalised Hermite and Laguerre polynomials are limiting cases of the generalised Jacobi polynomials (see e.g. [1]) this would imply the equivalence also in these two cases. If established, this result would in these cases imply a natural orthogonality for the reduced eigenfunctions in Theorem 2. At this point these statements are only conjectures and we hope to return to them elsewhere.

\subsection{Deformed Calogero-Sutherland models}

There exist an interesting deformation of Calogero-Sutherland type models [4, 21, 22], defined by the following class of differential operators in two sets of variables $x=\left(x_{1}, \ldots, x_{N}\right)$ and $\tilde{x}=\left(\tilde{x}_{1}, \ldots, \tilde{x}_{\tilde{N}}\right)$ :

$$
\begin{aligned}
H_{N, \tilde{N}}= & \sum_{j=1}^{N}\left(-\frac{\partial^{2}}{\partial x_{j}^{2}}+V\left(x_{j}\right)\right)-\sum_{J=1}^{\tilde{N}} \kappa\left(-\frac{\partial^{2}}{\partial \tilde{x}_{J}^{2}}+\tilde{V}\left(\tilde{x}_{J}\right)\right) \\
& +\kappa(\kappa-1) \sum_{j<k} W\left(x_{j}, x_{k}\right)+(1-\kappa) \sum_{j, K} W\left(x_{j}, \tilde{x}_{K}\right)+\frac{\kappa-1}{\kappa} \sum_{J<K} W\left(\tilde{x}_{J}, \tilde{x}_{K}\right),
\end{aligned}
$$


where the potential function $\tilde{V}$ is obtained from $V$ by a simple parameter shift; see [8]. They provide a natural generalisation of the models discussed in the previous section, in that they also have polynomial eigenfunctions. To be more precise, they have eigenfunctions which can be labelled by partitions $\lambda=\left(\lambda_{1}, \lambda_{2}, \ldots\right)$ such that $\lambda_{N+1} \leq \tilde{N}$ (see [22]), and are of the form

$$
\Psi_{\lambda}(x, \tilde{x})=\Psi_{0}(x, \tilde{x}) P_{\lambda}\left(z\left(x_{1}\right), \ldots, z\left(x_{N}\right), z\left(\tilde{x}_{1}\right), \ldots, z\left(\tilde{x}_{\tilde{N}}\right)\right)
$$

where the function $\Psi_{0}$ is given by

$$
\Psi_{0}(x, \tilde{x})=\prod_{j=1}^{N} \psi_{0}\left(x_{j}\right) \prod_{J=1}^{\tilde{N}} \tilde{\psi}_{0}\left(\tilde{x}_{J}\right) \frac{\prod_{j<k}\left(z\left(x_{k}\right)-z\left(x_{j}\right)\right)^{\kappa} \prod_{J<K}\left(z\left(\tilde{x}_{K}\right)-z\left(\tilde{x}_{J}\right)\right)^{1 / \kappa}}{\prod_{j, K}\left(z\left(\tilde{x}_{K}\right)-z\left(x_{j}\right)\right)},
$$

and the $P_{\lambda}$ are polynomials in the variables $z_{j}=z\left(x_{j}\right)$ and $\tilde{z}_{J}=z\left(\tilde{x}_{J}\right)$. They are however no longer symmetric under permutations of all variables but only under permutations restricted to the $x_{j}$ or the $\tilde{x}_{J}$. In addition, they obey the condition

$$
\left(\frac{\partial}{\partial z_{j}}+\kappa \frac{\partial}{\partial \tilde{z}_{J}}\right) P_{\lambda}=0
$$

on the hyperplanes $z_{j}=\tilde{z}_{J}$, for all $j=1, \ldots, N$ and $J=1, \ldots, \tilde{N}$. The corresponding algebra of polynomials has been extensively studied by Sergeev and Veselov [21, 22].

In [8] we also construct explicit series representations for the reduced eigenfunctions $P_{\lambda}$ of these 'deformed' Calogero-Sutherland type models. The construction is analogous to the one discussed in previous sections, with the difference that the reduced eigenfunctions now are expressed in a set of polynomials $f_{n, \tilde{n}},(n, \tilde{n}) \in \mathbb{Z}^{N+\tilde{N}}$, defined through the expansion of their generating function

$$
\begin{gathered}
\frac{\prod_{j<k}\left(1-\frac{y_{j}}{y_{k}}\right)^{\kappa} \prod_{J<K}\left(1-\frac{\tilde{y}_{J}}{\tilde{y}_{K}}\right)^{1 / \kappa}}{\prod_{j, K}\left(1-\frac{y_{j}}{\tilde{y}_{K}}\right)} \frac{\prod_{j, K}\left(1-\frac{x_{j}}{\tilde{y}_{K}}\right) \prod_{J, k}\left(1-\frac{\tilde{x}_{J}}{y_{k}}\right)}{\prod_{j, k}\left(1-\frac{x_{j}}{y_{k}}\right)^{\kappa} \prod_{J, K}\left(1-\frac{\tilde{x}_{J}}{\tilde{y}_{K}}\right)^{1 / \kappa}} \\
=\sum_{(n, \tilde{n}) \in \mathbb{Z}^{N+\tilde{N}}} f_{n, \tilde{n}}(x, \tilde{x}) y^{-n} \tilde{y}^{-\tilde{n}},
\end{gathered}
$$

valid for $\left|\tilde{y}_{\tilde{N}}\right|>\cdots\left|\tilde{y}_{1}\right|>\left|y_{N}\right|>\cdots>\left|y_{1}\right|>\max _{j, J}\left(\left|x_{j}\right|,\left|\tilde{x}_{J}\right|\right)$. We mention finally that the number of variables $x_{j}$ and $\tilde{x}_{J}$ as well as $y_{j}$ and $\tilde{y}_{J}$ may be chosen differently in the definition of these polynomials $f_{n, \tilde{n}}$, thus allowing for a number of series representations to be obtained for the same reduced eigenfunction, an aspect of our construction which is further discussed in [8].

\section{Acknowledgements}

I would like to thank Edwin Langmann and the three referees for a number of helpful comments on the manuscript. Financial support from the Knut and Alice Wallenberg foundation and the European Union through the FP6 Marie Curie RTN ENIGMA (Contract number MRTN-CT2004-5652) is also gratefully acknowledged. 


\section{References}

[1] Baker T.H., Forrester P.J., The Calogero-Sutherland model and generalized classical polynomials, Comm. Math. Phys. 188 (1997), 175-216, solv-int/9608004.

[2] Calogero F., Groundstate of a one-dimensional N-body system, J. Math. Phys. 10 (1969), 2197-2200.

[3] Calogero F., Solution of the one-dimensional $N$ body problems with quadratic and/or inversely quadratic pair potentials, J. Math. Phys. 12 (1971), 419-436.

[4] Chalykh O., Feigin M., Veselov A., New integrable generalizations of Calogero-Moser quantum problems, J. Math. Phys. 39 (1998), 695-703.

[5] Desrosiers P., Lapointe L., Mathieu P., Explicit formulas for the generalized Hermite polynomials in superspace, J. Phys. A: Math. Gen. 37 (2004), 1251-1268, hep-th/0309067.

[6] Gómez-Ullate D., González-López A., Rodríguez M.A., New algebraic quantum many-body problems, J. Phys. A: Math. Gen. 33 (2000), 7305-7335, nlin.SI/0003005.

[7] Hallnäs M., Langmann E., Explicit formulae for the eigenfunctions of the $N$-body Calogero model, J. Phys. A: Math. Gen. 39 (2006), 3511-3533, math-ph/0511040.

[8] Hallnäs M., Langmann E., Quantum Calogero-Sutherland type models and generalised classical polynomials, in preparation.

[9] Knop F., Sahi S., A recursion and a combinatorial formula for Jack polynomials, Invent. Math. 128 (1997), 9-22, q-alg/9610016.

[10] Kuznetsov V.B., Mangazeev V.V., Sklyanin E.K., Q-operator and factorised separation chain for Jack polynomials, Indag. Math. (N.S.) 14 (2003), 451-482, math.CA/0306242.

[11] Langmann E., Algorithms to solve the (quantum) Sutherland model, J. Math. Phys. 42 (2001), 4148-4157, math-ph/0104039.

[12] Langmann E., A method to derive explicit formulas for an elliptic generalization of the Jack polynomials, in Proceedings of the Conference "Jack, Hall-Littlewood and Macdonald Polynomials" (September 23-26, 2003, Edinburgh), Editors V.B. Kuznetsov and S. Sahi, Contemp. Math. 417 (2006), 257-270, math-ph/0511015.

[13] Lassalle M., Polynômes de Jacobi généralisés, C. R. Math. Acad. Sci. Paris 312 (1991), 425-428.

[14] Lassalle M., Polynômes de Laguerre généralisés, C. R. Math. Acad. Sci. Paris 312 (1991), 725-728.

[15] Lassalle M., Polynômes de Hermite généralisés, C. R. Math. Acad. Sci. Paris 313 (1991), 579-582.

[16] Lassalle M., Schlosser M., Inversion of the Pieri formula for MacDonald polynomials, Adv. Math. 202 (2006), 289-325, math.CO/0402127.

[17] MacDonald I.G., Symmetric functions and Hall polynomials, 2nd ed., Oxford University Press, 1995.

[18] MacDonald I.G., Hypergeometric functions, unpublished manuscript.

[19] Olshanetsky M.A., Perelomov A.M., Quantum integrable systems related to Lie algebras, Phys. Rep. 94 (1983), 313-404.

[20] Reed M., Simon B., Methods of modern mathematical physics. II. Fourier analysis, self-adjointness, Academic Press, 1975.

[21] Sergeev A.N., Veselov A.P., Deformed quantum Calogero-Moser problems and Lie superalgebras, Comm. Math. Phys. 245 (2004), 249-278, math-ph/0303025.

[22] Sergeev A.N., Veselov A.P., Generalised discriminants, deformed Calogero-Moser-Sutherland operators and super-Jack polynomials, Adv. Math. 192 (2005), 341-375, math-ph/0307036.

[23] Sutherland B., Quantum many-body problem in one dimension: ground state, J. Math. Phys. 12 (1971), $246-250$.

[24] Sutherland B., Exact results for a quantum many-body problem in one dimension: II, Phys. Rev. A 5 (1972), $1372-1376$.

[25] Szegö G., Orthogonal polynomials, American Mathematical Society, 1939.

[26] van Diejen J.F., Confluent hypergeometric orthogonal polynomials related to the rational quantum Calogero system with harmonic confinement, Comm. Math. Phys. 188 (1997), 467-497, q-alg/9609032.

[27] Lapointe L., Morse J., van Diejen J.F., Determinantal construction of orthogonal polynomials associated with root systems, Compos. Math. 140 (2004), 255-273, math.CO/0303263. 